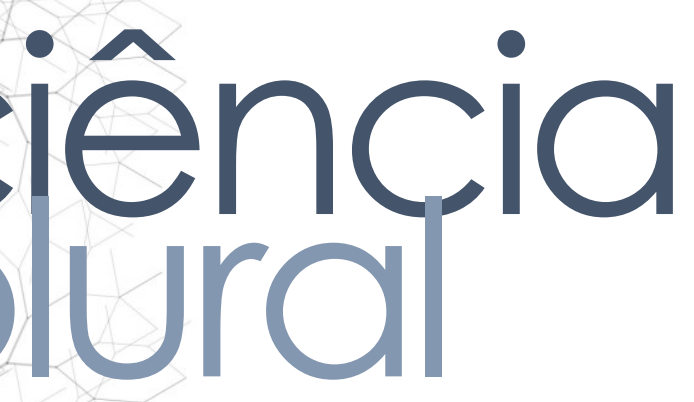

\title{
AVALIAÇÃO DO DESEMPENHO DOS CENTROS DE ESPECIALIDADES ODONTOLÓGICAS DO SERIDÓ POTIGUAR NO PERÍODO DE 2012 A 2017
}

Performance evaluation of dental care specialties centers of the Serido Potiguar from 2012 to 2017

Evaluación del desempeño de los Centros de Especialidades Odontológicas en Seridó Potiguar de 2012 a 2017

Thereza Raquel Garcia Silva Correia • Universidade Federal do Rio Grande do Norte• E-mail: therezargsc@gmail.com

Daniela Mendes da Veiga Pessoa • Universidade do Estado do Rio Grande do Norte • E-mail: danielamvp@yahoo.com.br

Autora responsável pela correspondência:

Thereza Raquel Garcia Silva Correia • E-mail: therezargsc@gmail.com 


\section{RESUMO}

Introdução: A política nacional de saúde bucal foi elaborada tendo como um dos objetivos a ampliação e qualificação da atenção especializada com a criação dos Centros de Especialidades Odontológicas (CEO). Para isso, ações de monitoramento de produção se fazem necessárias na busca de uma maior efetividade das ações produzidas. Objetivo: avaliar o desempenho dos dois CEO instalados nos municípios pólos de atenção à saúde na região Seridó Potiguar, a partir do cumprimento das metas de procedimentos clínicos especializados propostas pela Portaria GM N ${ }^{\circ}$ 1.464/2011. Método: O estudo é do tipo quantitativo, descritivo e exploratório.Para a análise de desempenho dos serviços odontológicos especializados, foi calculado o índice Cumprimento Global de Metas (CGM) para os procedimentos em endodontia, periodontia e cirurgia oral menor, extraídos do Sistema de Informação Ambulatorial do SUS (SIA-SUS). Resultados: O CEO de Parelhas conseguiu atingir as metas em Periodontia nos anos de 2013, 2015 e 2016, apresentando desempenho regular nesses anos e desempenho ruim nos demais. O CEO de Currais Novos atingiu apenas a meta de Periodontia em 2012, caracterizando um desempenho regular nesse ano e demonstrando nos outros anos desempenho ruim. Conclusões: Os municípios pesquisados apresentaram desempenho insatisfatório no período avaliado, variando de ruim à regular, onde possivelmente, fatores como cobertura populacional de equipes de saúde bucal na atenção básica e falhas na referência e contra referência para a atenção secundária apontam uma desorganização dos serviços nos diferentes níveis de atenção, causando a falta da integralidade do cuidado.

Palavras-Chave: Saúde Bucal. Assistência Odontológica Integral. Avaliação de Serviços de Saúde.

\section{ABSTRACT}

Introduction: The national oral health policy was intended for increase and qualify the specialassistance based on the criation of specialized dental centers (CEOs). In order to do so, monitoring and evaluation actions are important to provide greater effectiveness. Objective: measure the performance of CEOs belonging in two of regional clusters of Rio Grande do Norte state, analyzing the goals established for Ordinance GM No. 1.464/2011. Methodology: This study hás descriptive, quantitative and exploratory aspects. The methodology for specialized oral health assistance analysis is to calculate the Global Milestone Targets index (CGM) which considers endodontic, periodontics and oral surgery treatment procedures registered on the Ambulatory Information Portal of SUS. Results: The CEO/Parelhas district archive the periodontics goals of 2013, 2015 and 2016, which demonstrated regular performance in only these years. The CEO/Currais Novos district achieves only periodontics goals in 2012, which demonstrated regular performance in only this year nd poor performance in others years. Conclusions: The two regional districts have oor performance throughout the period evaluated, where factors like the low mand of oral health staffs and failures of treatments routes indicates a need for an gent measures to organize and integrate the oral health assistance network.

words: Oral Health. Dental Care. Health Services Evaluation. 


\section{ciência
piural}

\section{RESUMEN}

Introducción: La política nacional de salud oral se diseñó con el objetivo de expandir y calificar la atención especializada con la creación de los Centros de Especialidades Odontológicas (CEO). Para esto, el monitoreo de la producción es necesario en busca de una mayor efectividad de las acciones producidas. Objetivo: evaluar el desempeño de los CEO ubicados en los municipios que son polos de la atención de la salud en la región del Seridó Potiguar, a partir del cumplimiento de los objetivos de los procedimientos clínicos especializados propuestos por la Ordenanza GM No. 1.464 / 2011. Metodología: El estudio es cuantitativo, descriptivo y exploratorio. Para el análisis del desempeño de los servicios odontológicos especializados, se calculó el Índice Global de Logro de Metas (CGM) para los procedimientos en endodoncia, periodoncia y cirugía oral menor, extraído del Sistema de Información Ambulatoria del SUS (SIA-SUS). Resultados: El CEO en Parelhas logró los objetivos en Periodoncia en los años 2013, 2015 y 2016, presentando un desempeño regular en estos años y un desempeño deficiente en los demás. El CEO en Currais Novos solo logró el objetivo en Periodoncia en 2012, presentando un desempeño regular en este año y mostrando un desempeño pobre en los otros años. Conclusiones: los municipios encuestados presentaron un desempeño insatisfactorio en el período evaluado, que varía de pobre para regular, donde posiblemente factores como la cobertura de la población de los equipos de salud oral en atención primaria y las fallas en la referencia y en la contra referencia para la atención secundaria indican una desorganización de los serviciosen los diferentes niveles de la atención, lo que provoca la falta de atención integral.

Palabras Clave: Salud Bucal. Atención Odontológica Integral. Investigación sobre Servicios de Salud. 


\section{Introdução}

A saúde bucal no Brasil vem passando por um processo de reorganização, no qual as ações de saúde bucal no Sistema Único de Saúde (SUS) estão voltadas para a promoção de saúde, prevenção de agravos, diagnóstico, tratamento e reabilitação desde a implantação da Política Nacional de Saúde - "Brasil Sorridente" em 20041,2,3.

O "Brasil Sorridente" foi fundamentado, epidemiologicamente, a partir do "Projeto SB Brasil 2003 - Condições de Saúde Bucal da População Brasileira", onde seus resultados apresentaram altos índices do número médio de dentes permanentes cariados, perdidos e obturados (CPO-D), com destaque para a perda dentária (28\% dos adultos não possuíam nenhum dente funcional em pelo menos uma arcada e $75 \%$ dos idosos não possuíam nenhum dente funcional) ${ }^{4,5}$.

Tais resultados levaram à criação da Política Nacional de Saúde Bucal - Brasil Sorridente e ao planejamento das ações em saúde bucal no país 4,6,7, lançado em prol da reestruturação da Saúde Bucal na Atenção Básica.

O Brasil Sorridente prioriza as Equipes de Saúde Bucal na Estratégia de Saúde da Família, a ampliação e qualificação da atenção especializada (especialmente com a implantação de Centros de Especialidades Odontológicas - CEO - e Laboratórios Regionais de Próteses Dentárias - LRPD) e a viabilização da adição de flúor nas estações de tratamento de águas de abastecimento público ${ }^{5}$.

Os Centros de Especialidades Odontológicas (CEO) são estabelecimentos de saúde cadastrados no Cadastro Nacional de Estabelecimentos de Saúde são (CNES) e classificados como Tipo Clínica Especializada/Ambulatório de Especialidade e devem ofertar serviços especializados de média complexidade, tais quais: diagnóstico bucal, com ênfase no diagnóstico e detecção do câncer bucal, periodontia especializada, cirurgia oral menor dos tecidos moles e duros, endodontia e atendimento aos portadores de necessidades especiais ${ }^{8}$. Para isso, é necessário que o município tenha instituído e estruturado bem a sua rede de Atenção Básica para que haja a adequada ferta em serviços de saúde bucal de modo integral e hierarquizado ${ }^{1,9}$.

Em 2011, o Ministério da Saúde (MS) apresentou a Portaria No 1.464 de 24 de Junho 2011 que visa à reorganização das práticas e à qualificação dos serviços oferecidos Saúde Bucal preconizada pela Política Nacional de Saúde Bucal e propõe o 
monitoramento de produção, ao estabelecer a análise de uma produção mínima mensal apresentada, a ser realizada nos CEO, verificada por meio dos Sistemas de Informação Ambulatorial do SUS - SIA/SUS'10.

Leva-se em consideração que o perfil epidemiológico em Saúde Bucal no Brasil está em progresso, de modo que a pesquisa SBBrasil 2010 apresentou melhoras com relação aos resultados de 2003, porém não atingiu as metas propostas pelo projeto e ainda apresenta índices inferiores aos preconizados para 2010 pela Organização Mundial de Saúde ${ }^{11,12}$.

Isso ocorre, principalmente, pela desigualdade relacionada ao acesso aos serviços odontológicos, à gravidade e a perda dentária precoce, caracterizando o endentulismo como um problema de saúde pública persistente no Brasil².

Nesse contexto, o Nordeste brasileiro lidera com os maiores indicadores sociais desfavoráveis, com elevados índices de deficiências na saúde bucal, agravando-se ainda mais ao comparar-se os índices de capitais para cidades do interior do país ${ }^{4,7}$.

Portanto, o presente estudo tem como objetivo realizar uma avaliação e monitoramento do desempenho dos Centros de Especialidades Odontológicas instalados na região Seridó, referente à IV Região de Saúde, do estado do Rio Grande do Norte (RN), nos municípios de Currais Novos e Parelhas, a partir do cumprimento das metas de procedimentos ambulatoriais propostas pela Portaria GM No 1.464/2011.

\section{Metodologia}

\section{Tipo de estudo}

O estudo realizado foi do tipo quantitativo, descritivo e exploratório.

\section{Cenário da pesquisa}

A pesquisa foi realizada na região do Seridó Potiguar, onde se concentra a IV Região de Saúde do estado do Rio Grande do Norte, que abrange os 24 municípios a eguir: Acari, Bodó, Cerro Corá, Caicó, Cruzeta, Currais Novos, Equador, Florânia, Ipueira, Jardim de Piranhas, Jardim do Seridó, Jucurutu, Lagoa Nova, Ouro Branco, Parelhas, São Fernando, São Vicente, São João do Sabugi, São José do Seridó, Santana do Seridó, Serra Negra do Norte, Carnaúba dos Dantas, Timbaúba dos 
Batistas e Tenente Laurentino Cruz. Contendo sua população estimada em 310.067 habitantes ${ }^{13}$.

\section{Critério amostral}

Os CEO selecionados para compor as unidades amostrais do presente estudo pertencem aos municípios pólos de atenção à saúde da Região Seridó do estado do Rio Grande do Norte. Como critérios de inclusão foram admitidos os municípios de Currais Novos e Parelhas, que possuem CEO do Tipo II, sendo o de Currais Novos habilitado em 2006, contendo quatro equipos odontológicos para oito cirurgiõesdentistas, com serviços de Radiologia e Laboratório Regional de Prótese Dentária (LRPD). O CEO em Parelhas foi habilitado em 2011, com apenas quatro cirurgiõesdentistas e não oferta o serviço de Radiologia14.

\section{Procedimentos de coleta de dados}

Foram utilizados dados secundários, provenientes do banco de dados do Sistema de Informação Ambulatorial do Sistema Único de Saúde (SIA-SUS), constituindo-se da produção realizada por cada CEO nas especialidades de Periodontia, Endodontia e Cirurgia Oral Menor. Também foram coletados dados referentes à cobertura das Equipes de Saúde Bucal (ESB) de cada município que estão disponíveis no Departamento de Informática do Sistema Único de Saúde (DATASUS); dados referentes à caracterização dos CEO (tipo de CEO, categoria que foi habilitado, data de habilitação, presença de LRPD e de serviço de Radiologia, número de equipos e de Cirurgiões-dentistas) encontrados no Cadastro Nacional de Estabelecimentos de Saúde (CNES). Além dos referidos dados, foram coletadas informações sócio demográficas (população, população estimada, Índice de Desenvolvimento Humano (IDH), Produto Interno Bruto (PIB) per capita e taxa de analfabetismo na população acima de 15 anos), extraídas do Instituto Brasileiro de Geografia e Estatística (IBGE) ${ }^{15}$.

Os dados de produção ambulatorial coletados são referentes ao período de 2012 a 2017. 


\section{Análise dos dados}

Os dados foram analisados a partir de estatística descritiva, cujo objetivo básico foi o de sintetizar os valores de mesma natureza (frequências absoluta e relativa percentual - dos procedimentos especializados em odontologia, em cada ano, por CEO) permitindo dessa forma uma visão global da variação desses valores. Nesse sentido, para a análise do desempenho da atenção de média complexidade, primeiramente foi calculada a média aritmética de procedimentos por especialidade odontológica, por ano, em cada CEO. O cálculo foi feito através da soma dos valores absolutos de cada procedimento por mês e dividido por 12 para estimar a produção mensal. Em seguida, a partir desse resultado, foi calculado o índice Cumprimento Global de Metas (CGM) baseado nas metas pré-estabelecidas pela Portaria GM N ${ }^{\circ}$ $1.464 / 2011^{10}$, através da fórmula a seguir:

\section{Cálculo do índice CGM:}

Quantidade total de procedimentos realizados durante $n$ meses de cada subgrupo de especialidades odontológicas X100

Número de procedimentos correspondente à meta deste subgrupo por $\mathbf{n}$ meses

A meta é considerada atingida quando o valor do CGM for igual ou maior que 100\%. A avaliação de desempenho pelo CGM foi modificada ao ser retirado do estudo o subgrupo de procedimentos básicos por conter procedimentos em comum com a atenção básica, o que tem influência sobre as atividades da atenção secundária.

Sendo assim, foram estabelecidas três metas a serem cumpridas: procedimentos em Endodontia, Periodontia e Cirurgia Oral Menor, de modo que os serviços tiveram seus desempenhos classificados em: ruim (quando não cumpriram nenhuma meta), regular (quando cumpriram apenas uma meta), bom (quando cumpriram duas metas) ótimo (quando cumpriram as três metas) ${ }^{9}$. (Tabela 1) 
Tabela 1: Metas de procedimentos especializados em saúde bucal por mês/ano de acordo com o tipo de CEO. Caicó-RN, 2018

\begin{tabular}{cccc}
\hline $\begin{array}{c}\text { Procedimentos } \\
\text { conforme } \\
\text { Especialidades }\end{array}$ & $\begin{array}{c}\text { Metas para CEO Tipo I } \\
\text { (por mês/ano) }\end{array}$ & $\begin{array}{c}\text { Metas para CEO Tipo } \\
\text { II (por mês/ano) }\end{array}$ & $\begin{array}{c}\text { Metas para CEO Tipo } \\
\text { III (por mês/ano) }\end{array}$ \\
\hline $\begin{array}{c}\text { Procedimentos de } \\
\text { Periodontia }\end{array}$ & $60 / 720$ & $90 / 1080$ & $150 / 1800$ \\
$\begin{array}{c}\text { Procedimentos de } \\
\text { Cirurgia Oral } \\
\text { Procedimentos de } \\
\text { Endodontia }\end{array}$ & $80 / 960$ & $90 / 1080$ & $170 / 2040$ \\
\hline
\end{tabular}

Fonte: Portaria No 1.464 de 24 de Junho de 2011, Ministério da Saúde.

\section{Resultados}

O CEO de Parelhas conseguiu atingir as metas do subgrupo de Periodontia nos anos de 2013, 2015 e 2016. Sendo assim, apresentando desempenho regular nesses anos e desempenho ruim nos demais anos. O CEO de Currais Novos conseguiu atingir, somente, a meta de Periodontia em 2012, demonstrando um desempenho regular em 2012 e nos demais anos, desempenho ruim. (Tabela 2)

Tabela 2: Média mensal de procedimentos e índice Cumprimento Global de Metas por especialidade em cada CEO. Caicó-RN, 2018

\begin{tabular}{|c|c|c|c|c|c|}
\hline & Ano & $\begin{array}{l}\text { Média mensal de } \\
\text { procedimentos }\end{array}$ & $\begin{array}{l}\text { Cumprimento } \\
\text { de metas }\end{array}$ & $\begin{array}{c}\text { Cumprimento } \\
\text { global }\end{array}$ & $\begin{array}{c}\text { Classificação } \\
\text { de } \\
\text { desempenho }\end{array}$ \\
\hline \multirow{7}{*}{ Currais Novos } & 2012 & $\begin{array}{l}\text { Periodontia: } 113,83 \\
\text { Endodontia: } 10,83 \\
\text { Cirurgia oral: } 38,00\end{array}$ & $\begin{array}{l}189,71 \% \\
30,94 \% \\
47,50 \%\end{array}$ & $\begin{array}{l}\text { Sim } \\
\text { Não } \\
\text { Não }\end{array}$ & Regular \\
\hline & 2013 & $\begin{array}{l}\text { Periodontia: } 42,00 \\
\text { Endodontia: } 32,16 \\
\text { Cirurgia oral: } 36,91\end{array}$ & $\begin{array}{l}70,00 \% \\
53,60 \% \\
46,13 \%\end{array}$ & $\begin{array}{l}\text { Não } \\
\text { Não } \\
\text { Não }\end{array}$ & Ruim \\
\hline & 2014 & $\begin{array}{l}\text { Periodontia: 56,25 } \\
\text { Endodontia: } 26,16 \\
\text { Cirurgia oral: } 31,16\end{array}$ & $\begin{array}{l}62,50 \% \\
43,60 \% \\
35,11 \%\end{array}$ & $\begin{array}{l}\text { Não } \\
\text { Não } \\
\text { Não }\end{array}$ & Ruim \\
\hline & 2015 & $\begin{array}{l}\text { Periodontia: 41,75 } \\
\text { Endodontia: 15,41 } \\
\text { Cirurgia oral: } 17,83\end{array}$ & $\begin{array}{l}46,38 \% \\
25,68 \% \\
22,28 \%\end{array}$ & $\begin{array}{l}\text { Não } \\
\text { Não } \\
\text { Não }\end{array}$ & Ruim \\
\hline & 2016 & $\begin{array}{l}\text { Periodontia: } 10,66 \\
\text { Endodontia: } 13,16 \\
\text { Cirurgia oral: } 12,08\end{array}$ & $\begin{array}{l}11,84 \% \\
21,93 \% \\
15,10 \%\end{array}$ & $\begin{array}{l}\text { Não } \\
\text { Não } \\
\text { Não }\end{array}$ & Ruim \\
\hline & 2017 & $\begin{array}{l}\text { Periodontia: } 31,66 \\
\text { Endodontia: 11,33 } \\
\text { Cirurgia oral: } 18,08\end{array}$ & $\begin{array}{l}35,17 \% \\
18,83 \% \\
22,60 \%\end{array}$ & $\begin{array}{l}\text { Não } \\
\text { Não } \\
\text { Não }\end{array}$ & Ruim \\
\hline & 2012 & $\begin{array}{l}\text { Periodontia: } 48,33 \\
\text { Endodontia: } 15,50 \\
\text { Cirurgia oral: } 22,58\end{array}$ & $\begin{array}{l}53,70 \% \\
25,83 \% \\
28,22 \%\end{array}$ & $\begin{array}{l}\text { Não } \\
\text { Não } \\
\text { Não }\end{array}$ & Ruim \\
\hline
\end{tabular}




\section{ciência plural}

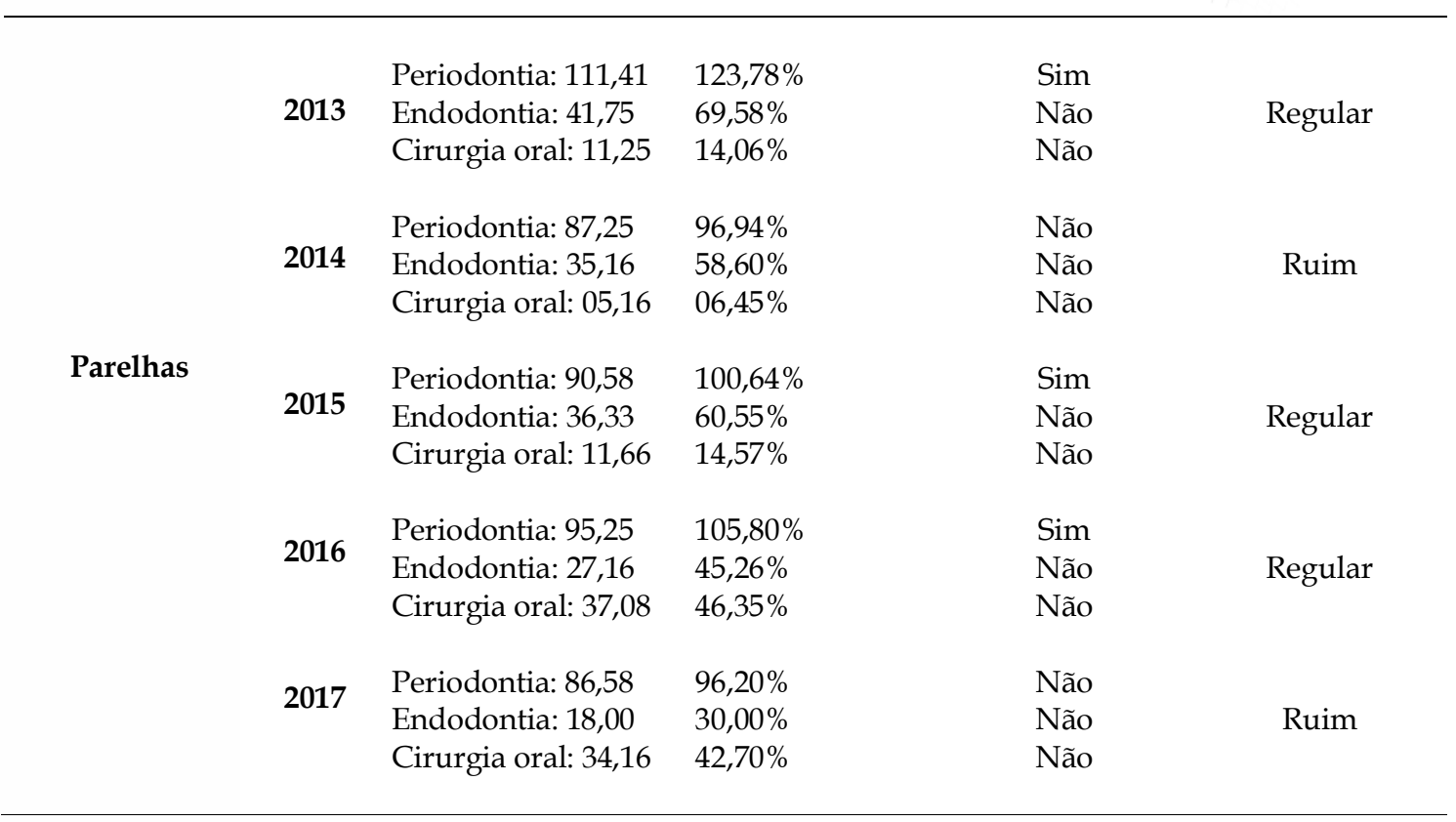

Em relação aos procedimentos por subgrupos em ambos os municípios, no subgrupo dos procedimentos endodônticos, a obturação em dente permanente (com uma, duas, três ou mais raízes) foi o procedimento mais realizado.

No subgrupo dos procedimentos de cirurgia oral menor, em Currais Novos (Tabela 3) o mais realizado foi a remoção de dente retido (incluso/impactado) e em Parelhas (Tabela 4), a curetagem periapical esteve à frente dos procedimentos realizados dentro dessa especialidade.

Tabela 3: Procedimentos anuais por especialidade realizados no município de Currais Novos entre 2012 e 2017. Caicó-RN, 2018

\begin{tabular}{|c|c|c|c|c|c|c|c|c|c|c|c|c|c|}
\hline \multirow{2}{*}{ Município } & \multirow{2}{*}{ Procedimento } & \multirow{2}{*}{$\begin{array}{r}2012 \\
\text { n }\end{array}$} & \multicolumn{3}{|c|}{2013} & \multicolumn{2}{|l|}{2014} & \multicolumn{2}{|c|}{2015} & \multicolumn{2}{|l|}{2016} & \multicolumn{2}{|l|}{2017} \\
\hline & & & $\%$ & $\mathbf{n}$ & $\%$ & $\mathbf{n}$ & $\%$ & $\mathbf{n}$ & $\%$ & $\mathrm{n}$ & $\%$ & $\mathrm{n}$ & $\%$ \\
\hline \multirow[t]{6}{*}{ Currais Novos } & ENDODONTIA & & & & & & & & & & & & \\
\hline & $\begin{array}{l}\text { Obturação de dente } \\
\text { decíduo }\end{array}$ & 0 & 0 & 73 & 18,9 & 11 & 3,51 & 7 & 3,79 & 1 & 0,64 & 18 & 13,2 \\
\hline & $\begin{array}{l}\text { Obturação de dente } \\
\text { permanente } \\
\text { uni/birradicular e com } 3 \\
\text { ou mais raízes }\end{array}$ & 77 & 59,2 & 224 & 224 & 295 & 94 & 178 & 96,2 & 149 & 94,3 & 115 & 4,55 \\
\hline & $\begin{array}{l}\text { Retratamento } \\
\text { endodôntico em dente } \\
\text { permanente } \\
\text { uni/birradicular e com } 3 \\
\text { ou mais raízes }\end{array}$ & 12 & 9,23 & 5 & 1,29 & 7 & 2,23 & 0 & 0 & 0 & 0 & 3 & 2,2 \\
\hline & $\begin{array}{l}\text { Selamento de } \\
\text { perfuração radicular }\end{array}$ & 41 & 31,5 & 84 & 21,8 & 1 & 0,31 & 0 & 0 & 8 & 5,06 & 0 & 0 \\
\hline & TOTAL & 130 & 100 & 386 & 100 & 314 & 100 & 185 & 100 & 158 & 100 & 136 & 100 \\
\hline
\end{tabular}




\section{PERIODONTIA}

Raspagem coronoradicular (por sextante)

Gengivectomia (por sextante)

Gengivoplastia (por sextante)

Tratamento cirúrgico periodontal (por sextante)

TOTAL

\section{CIRURGIA ORAL} MENOR

Biópsia (glândula salivar, osso do crânio e face, e tecidos moles da boca)

Remoção de dente retido

(incluso/impactado)

Correção de irregularidades de rebordo alveolar e tuberosidade maxilar Curetagem periapical TOTAL

\begin{tabular}{|c|c|c|c|c|c|c|c|c|c|}
\hline 1344 & 98,4 & 757 & 96,6 & 648 & 96 & 478 & 94,9 & 127 & 9,2 \\
\hline 20 & 1,47 & 22 & 22 & 27 & 4 & 20 & 3,96 & ( & \\
\hline 2 & 0,15 & 5 & 0,63 & 0 & 0 & 0 & 0 & 1 & 0,7 \\
\hline 0 & 0 & 0 & 0 & 0 & 0 & 6 & 1,19 & ( & \\
\hline 1366 & 100 & 784 & 100 & 675 & 100 & 504 & 100 & 128 & 10 \\
\hline 0 & 0 & 3 & 0,68 & 1 & 0,28 & 1 & 0,48 & 6 & 4,1 \\
\hline 420 & 92,1 & 339 & 76,5 & 273 & 73 & 179 & 83,6 & 138 & 95 \\
\hline 12 & 2,63 & 12 & 2,71 & 9 & 2,4 & 0 & 0 & 7 & 1 \\
\hline 24 & 5,26 & 89 & 20,1 & 91 & 24,3 & 34 & 15,9 & ( & \\
\hline 456 & 100 & 443 & 100 & 374 & 100 & 214 & 100 & 14 & 10 \\
\hline
\end{tabular}

Fonte:Portal da Saúde (DATASUS). Informações de Saúde (TABNET), Ministério da Saúde.

Tabela 4: Procedimentos anuais por especialidade realizados no município de Parelhas entre 2012 e 2017. Caicó-RN, 2018.

\begin{tabular}{|c|c|c|c|c|c|c|c|c|c|c|c|c|c|}
\hline \multirow{3}{*}{ Município } & \multirow{3}{*}{ Procedimento } & \multicolumn{12}{|c|}{ Ano } \\
\hline & & \multicolumn{2}{|l|}{2012} & \multicolumn{2}{|l|}{2013} & \multicolumn{2}{|l|}{2014} & \multicolumn{2}{|l|}{2015} & \multicolumn{2}{|l|}{2016} & \multicolumn{2}{|l|}{2017} \\
\hline & & $\mathbf{N}$ & $\%$ & $\mathbf{n}$ & $\%$ & $\mathbf{n}$ & $\%$ & $\mathbf{n}$ & $\%$ & $\mathbf{n}$ & $\%$ & $\mathbf{n}$ & $\%$ \\
\hline \multirow[t]{9}{*}{ Parelhas } & ENDODONTIA & & & & & & & & & & & & \\
\hline & $\begin{array}{l}\text { Obturação de dente } \\
\text { decíduo }\end{array}$ & 18 & 9,68 & 7 & 1,39 & 25 & 5,92 & 0 & 0 & 1 & 0,39 & 0 & 0 \\
\hline & $\begin{array}{l}\text { Obturação de dente } \\
\text { permanente } \\
\text { uni/birradicular e com } 3 \\
\text { ou mais raízes }\end{array}$ & 136 & 73,1 & 466 & 3,02 & 367 & 87 & 436 & 100 & 313 & 96 & 212 & 98,2 \\
\hline & $\begin{array}{l}\text { Retratamento } \\
\text { endodôntico em dente } \\
\text { permanente } \\
\text { uni/birradicular e com } 3 \\
\text { ou mais raízes }\end{array}$ & 16 & 8,6 & 28 & 5,59 & 30 & 7,12 & 0 & 0 & 12 & 3,6 & 4 & 1,85 \\
\hline & $\begin{array}{l}\text { Selamento de perfuração } \\
\text { radicular }\end{array}$ & 16 & 8,6 & 0 & 0 & 0 & 0 & 0 & 0 & 0 & 0 & 0 & 0 \\
\hline & $\begin{array}{l}\text { TOTAL } \\
\text { PFRIODONTIA }\end{array}$ & 186 & 100 & 501 & 100 & 422 & 100 & 436 & 100 & 326 & 100 & 216 & 100 \\
\hline & PERIODONTIA & & & & & & & & & & & & \\
\hline & $\begin{array}{l}\text { Raspagem corono- } \\
\text { radicular (por sextante) }\end{array}$ & 502 & 6,55 & 1282 & 95,9 & 1039 & 99,2 & 1060 & 97,5 & 1141 & 99,8 & 1038 & 99,9 \\
\hline & $\begin{array}{l}\text { Gengivectomia (por } \\
\text { sextante) }\end{array}$ & 47 & 8,1 & 13 & 0,97 & 4 & 0,38 & 12 & 1,1 & 0 & 0 & 0 & 0 \\
\hline
\end{tabular}




\begin{tabular}{|c|c|c|c|c|c|c|c|c|c|c|c|c|}
\hline $\begin{array}{l}\text { Gengivoplastia (por } \\
\text { sextante) }\end{array}$ & 28 & 4,83 & 11 & 0,83 & 1 & 0,1 & 7 & 0,65 & 1 & 0,08 & 1 & 0,1 \\
\hline $\begin{array}{l}\text { Tratamento cirúrgico } \\
\text { periodontal (por } \\
\text { sextante) }\end{array}$ & 3 & 0,52 & 31 & 2,32 & 3 & 0,29 & 8 & 0,74 & 1 & 0,08 & 0 & 0 \\
\hline TOTAL & 580 & 100 & 1337 & 100 & 1047 & 100 & 1087 & 100 & 1143 & 100 & 1039 & 100 \\
\hline $\begin{array}{l}\text { CIRURGIA ORAL } \\
\text { MENOR }\end{array}$ & & & & & & & & & & & & \\
\hline $\begin{array}{l}\text { Biópsia (glândula } \\
\text { salivar, osso do crânio e } \\
\text { face, e tecidos moles da } \\
\text { boca) }\end{array}$ & 0 & 0 & 0 & 0 & 0 & 0 & 0 & 0 & 0 & 0 & 0 & 0 \\
\hline $\begin{array}{l}\text { Remoção de dente retido } \\
\text { (incluso/impactado) }\end{array}$ & 1 & 0,37 & 49 & 36,3 & 53 & 85,5 & 111 & 79,3 & 172 & 38,7 & 136 & 33,2 \\
\hline $\begin{array}{l}\text { Correção de } \\
\text { irregularidades de } \\
\text { rebordo alveolar e } \\
\text { tuberosidade maxilar }\end{array}$ & 7 & 2,58 & 27 & 20 & 3 & 4,84 & 7 & 5 & 3 & 0,67 & 4 & 0,97 \\
\hline Curetagem periapical & 263 & 97,1 & 59 & 43,7 & 6 & 9,68 & 22 & 15,7 & 270 & 60,7 & 270 & 65,9 \\
\hline TOTAL & 271 & 100 & 135 & 100 & 62 & 100 & 140 & 100 & 445 & 100 & 410 & 100 \\
\hline
\end{tabular}

Fonte: Portal da Saúde (DATASUS). Informações de Saúde (TABNET), Ministério da Saúde.

Concernente aos dados sócio demográficos, os municípios possuem dados como, IDH, renda per capita e taxa de analfabetismo em maiores de 15 anos, muito parecidos, divergindo apenas na população, onde Currais Novos tem o dobro de habitantes que há em Parelhas e na cobertura das ESB, em que Currais Novos tem quase 100\% de sua população coberta e Parelhas não atingiu nem metade de sua população com cobertura de ESB. ${ }^{16,17}$ (Tabela 5)

Tabela 5:Caracterização sócio demográfica e cobertura populacional de Equipe de Saúde Bucal dos municípios de Currais Novos e Parelhas/RN. Caicó-RN, 2018

\begin{tabular}{lllllll}
\hline Município & $\begin{array}{l}\text { População } \\
\mathbf{( 2 0 1 0 ) *}^{*}\end{array}$ & $\begin{array}{l}\text { População } \\
\text { estimada } \\
\text { para 2017* }\end{array}$ & $\begin{array}{l}\text { IDH } \\
(\mathbf{2 0 1 0})^{*}\end{array}$ & $\begin{array}{l}\text { PIB per } \\
\text { capita (2015)* }\end{array}$ & $\begin{array}{l}\text { Taxa de } \\
\text { analfabetismo na } \\
\text { população acima dos } \\
\mathbf{1 5} \text { anos (2010)* }\end{array}$ & $\begin{array}{l}\text { Cobertura ESB } \\
(\mathbf{2 0 1 4})^{* *}\end{array}$ \\
\hline $\begin{array}{l}\text { Currais } \\
\text { Novos } \\
\text { Parelhas }\end{array}$ & 42.652 & 45.228 & 0,691 & $\mathrm{R} \$ 14.042,61$ & $16,94 \%$ & $98,03 \%$ \\
\hline
\end{tabular}

*Fonte: Instituto Brasileiro de Geografia e Estatística.

**Fonte:Portal da Saúde (DATASUS). Informações de Saúde (TABNET), Ministério da Saúde. 


\section{Discussão}

O SIA-SUS16, apesar de não fornecer dados de vigilância epidemiológica e da natureza da informação correr o risco de sofrer subnotificação, falta de atualização e erros na categorização dos procedimentos, esse sistema de informação é uma ferramenta de fundamental importância na avaliação das políticas de saúde bucal, além de possibilitar o acesso a informações como à oferta de serviços ambulatoriais, com base em indicadores de cobertura ${ }^{17}$.

No presente estudo, foi possível observar que o desempenho dos CEO de Currais Novos e Parelhas variou entre ruim e regular, respectivamente e frente à realidade do perfil de saúde bucal ser mais deficiente nas regiões Norte e Nordeste do país ${ }^{4,7}$, estudos que se propuseram a avaliar o desempenho dos serviços de atenção secundária em saúde bucal, nessas regiões, encontraram resultados semelhantes 2,9,12,18.

Um aspecto considerável para justificar o baixo desempenho dos Centros de Especialidades Odontológicas, é a questão da referência e contra referência que devem ocorrer de forma efetiva, para que a abordagem do tratamento seja integral e não haja barreiras entre os níveis de atenção. No I Encontro Nacional de Centro de Especialidades Odontológicas - CEO e Equipes de Saúde Bucal da Estratégia de Saúde da Família, segundo o Ministério da Saúde, foram discutidos alguns desafios enfrentados pela atenção secundária em saúde bucal, tais como: a demanda reprimida, a falta de estrutura e materiais, recursos humanos insuficientes e a desorganização do trabalho que refletem no prolongamento do tempo de espera para o atendimento odontológico especializado $^{6}$. Tais entraves, quando superados, estão diretamente relacionados com a satisfação do paciente, com a efetivação e conclusão do seu tratamento, pois podem evitar que um nível de atenção sirva de escape para o outro, ainda que seja sobrecarregado por demandas que não lhes competem. ${ }^{6}$

Diante dessa problemática, Figueiredo e colaboradores avaliaram o desempenho 22 CEO de Pernambuco durante o ano de 2006, de acordo com o Cumprimento obal de Metas, onde 31,8\% dos Centros de Especialidades Odontológicas resentaram o desempenho ruim, sendo o subgrupo dos procedimentos de cirurgia

menor o que teve o menor percentual do cumprimento de metas $(13,6 \%)^{18}$. tana e colaboradores realizaram uma análise da evolução desse desempenho em 
Recife (PE) num período de oito anos, entre 2000 e 2007, constatando de fato a insuficiência dos serviços especializados em saúde bucal e a dificuldade de acesso população para os mesmos. Através de uma tentativa de reorganizar o sistema de atenção em saúde bucal, a gestão municipal tentou infundir uma parte da assistência especializada nas Unidades Básicas de Saúde da Família, descaracterizando a proposta do funcionamento dos serviços prestados na Atenção Básica ${ }^{18,19}$.

Realidade semelhante foi encontrada no presente estudo, no qual o município de Currais Novos atingiu apenas uma meta (na especialidade de Periodontia em 2012). Enquanto, o município de Parelhas atingiu as metas de Periodontia nos anos de 2013, 2015 e 2016.

Em Currais Novos, alguns dos procedimentos odontológicos especializados são executados na Atenção Básica, tendo em vista que o procedimento de Periodontia mais realizado é a raspagem corono-radicular. Possivelmente, tal fato pode estar relacionado com a cobertura das Equipes de Saúde Bucal no município que apresenta 98,03\% da população coberta. O município de Parelhas concentra a assistência em saúde bucal especializada nos serviços de média complexidade por possuir apenas 48,39\% da população coberta por Equipes de Saúde Bucal, sendo esse um percentual insatisfatório, estando abaixo da média nacional e da região Nordeste de cobertura de Saúde Bucal na Atenção Básica, 52,26\% e 69,67\%, respectivamente ${ }^{20}$. Tal fato, trata-se de uma questão considerável em relação ao acúmulo das necessidades da população e direcionamento dos serviços da atenção básica para a secundária.

Também apresentando resultados de desempenho insatisfatórios, Santana e colaboradores analisaram 3 Centros de Especialidades Odontológicas, correspondentes a uma Região de Saúde do estado da Bahia, durante o ano de 2013. Todos tiveram seu desempenho classificados como ruim e, assim como Figueiredo et al. e Herkrath et al., foi observada uma relação diretamente proporcional entre o desempenho dos Centros de Especialidades Odontológicas e os indicadores ocioeconômicos. Achados semelhantes também foram observados no estudo de arros e colaboradores, no qual pesquisou as desigualdades na utilização e no acesso serviços odontológicos e concluiu que 39\% dos entrevistados que possuíam uma da familiar baixa, nunca foram ao dentista e que 43,3\% não foram atendidos no 
serviço público por falta de vaga. Tais achados podem caracterizar a relação entre o desempenho dos CEO e os índices socioeconômicos através da desigualdade e dificuldade de acesso $^{21}$.

Levando-se em consideração as avaliações de estudos anteriores ${ }^{12,18,21}$, a relação dos dados sócio demográficos e o cumprimento de metas insatisfatório dos CEO nos municípios estudados, pode-se levantar a hipótese de que haja uma relação positiva entre esses aspectos, visto que, embora o IDH de Currais Novos e Parelhas apresentem-se com valores ditos médios, ainda estão abaixo do IDH brasileiro $(0,718)$, demonstrando uma realidade de desenvolvimento humano dos municípios abaixo do estimado, sendo esse um elemento que infere nas variáveis de saúde, economia e educação, por exemplo, de forma que apresenta-se determinante na construção da consciência de suas necessidades ${ }^{13,22}$.

Observa-se que a busca por serviços de saúde acontece a partir do entendimento e reconhecimento de suas necessidades pelo paciente. Desenvolver essa percepção é um papel desempenhado, também, pela escola por ser esta um espaço privilegiado para se obter o alcance das informações de saúde. Para os municípios brasileiros que contam com o apoio do Programa de Saúde na Escola (PSE), tal programa pode influenciar fortemente e positivamente na prevenção e tratamento de doenças e agravos. Neste estudo, a taxa de analfabetismo na população acima de 15 anos, tanto em Currais Novos quanto em Parelhas estão acima da média nacional (9,6\%), o que poderia estar associado ao baixo desempenho dos $\mathrm{CEO}$ cem relação à procura por serviços odontológicos especializados: quanto maior o nível de escolaridade, mais ciente o indivíduo é das suas necessidades de saúde geral e bucal, assim buscando mais esses serviços ${ }^{13,22,23}$.

A partir da análise de dados do presente estudo, em relação aos procedimentos realizados no $\mathrm{CEO}$, pode-se levantar a questão de que no CEO de Parelhas não houve registro de realização de nenhum tipo de biópsia entre 2012 e 2017, apesar da região Seridó possuir uma alta incidência de radiação solar devido a sua localização e lima ${ }^{18,24}$. Fatores esses relacionados ao câncer bucal, que segundo os dados do stituto Nacional do Câncer (INCA) está entre os dez tipos de câncer mais incidentes imados para 2018, passando na frente do câncer de laringe, esôfago, leucemias e 
linfoma não Hodgkin. E a estimativa para 2018 é que 8,04 a cada 100.000 homens e 4,85 a cada 100.00 mulheres sejam acometidos pelo câncer na cavidade oral no estado do Rio Grande do Norte ${ }^{25}$.

O presente estudo permitiu a verificação do desempenho dos dois CEO instalados nos municípios polos de atenção à saúde na região Seridó Potiguar, a partir do cumprimento das metas de procedimentos clínicos especializados propostas pela Portaria GM No 1.464/2011. No entanto, por se tratar de uma metodologia descritiva, a pesquisa apresenta limitação, no sentido de não permitir a associação entre o desempenho do cumprimento de metas dos CEO com as características sócio demográficas e de assistência à saúde dos municípios dos quais fazem parte.

\section{Conclusões}

De acordo com os resultados encontrados, conclui-se que ambos os munícipios apresentaram desempenho insatisfatório no período avaliado, variando de ruim à regular, onde possivelmente, fatores como cobertura populacional de equipes de saúde bucal e falhas na realização da referência e contra referência demonstram uma desorganização do serviço em saúde entre os diferentes níveis de atenção. Como consequência, há falta de integralidade do cuidado, sobrecarregando uns serviços enquanto outros se tornam insuficientes em seu desempenho.

Além disso, indicadores socioeconômicos como IDH e taxa de analfabetismo na população acima dos 15 anos podem influenciar na percepção das necessidades de saúde pelo indivíduo, tornando a busca pela prevenção de doenças e agravos, e pelo tratamento odontológico um fator determinante na execução de procedimentos e, consequentemente, no cumprimento de metas dos serviços especializados em saúde bucal. 


\section{Referências}

1. Brasil MS. Passo a Passo das ações da Política Nacional de Saúde Bucal. Brasília: MS, 2016.

2. Freitas CHSM, Lemos GA, Pessoa TRRF, Araújo MF, Forte FDS. Atenção em saúde bucal: avaliação dos centros de especialidades odontológicas da Paraíba. Saúde Debate. 2016; 40(108): 131-143.

3. Marques PLP, Junior ARF, Saraiva RS, Carioca SPB, Pequeno LL, Vieira LJES. Análise de desempenho de centro de especialidades odontológicas regional do Ceará, Brasil. Saúde (Santa Maria). 2017; 43(3): 1-8.

4. Brasil MS. Projeto SB Brasil 2003: Condições de saúde bucal da população brasileira 2002-2003: resultados principais. Brasília: MS, 2004.

5. Brasil MS. Diretrizes da Política Nacional de Saúde Bucal. Brasília: MS, 2004.

6. Brasil MS. I Encontro Nacional de Centro de Especialidades Odontológicas - CEO e Equipes de Saúde Bucal da Estratégia de Saúde da Família. Brasília: MS, 2009.

7. Brasil MS. SB Brasil 2010: Pesquisa Nacional de Saúde Bucal: resultados principais. Brasília: MS, 2012.

8. Brasil MS. Portaria $n^{0} 1570$, de 29 de julho de 2004. Estabelece critérios, normas e requisitos para a implantação e credenciamento de centros de especialidades odontológicas e laboratórios regionais de próteses dentária. Diário Oficial da República Federativa do Brasil. Brasília: MS, 2004.

9. Santana DA, Santos LPS, Carvalho FS, Carvalho CAP. Desempenho dos centros de especialidades odontológicas de uma região de saúde da Bahia. Cad. Saúde Coletiva. 2015; 23(3): 261-267.

10. Brasil MS. Portaria $n^{\circ} 1464$, de 24 de junho de 2011. Altera o Anexo da Portaria $n^{\circ}$ 600/GM/MS, de 23 de março de 2006, que institui o financiamento dos centros de especialidades odontológicas (CEO). Diário Oficial da República Federativa do Brasil. Brasília: MS, 2011.

11. Roncalli AG, Côrtes MIS, Peres KG. Perfis epidemiológicos de saúde bucal no Brasil e os modelos de vigilância. Cad. Saúde Pública. 2012; 28(supl.): s58-s68. 
12. Herkrath FJ, Herkrath APCQ, Costa LNBS, Gonçalves MJF. Desempenho dos centros de especialidades odontológicas frente ao quadro sócio demográfico dos municípios do Amazonas, Brasil, 2009. Saúde Debate. 2013; 37(96): 148-158.

13. Instituto Brasileiro de Geografia e Estatística (IBGE). Censo 2010. Brasil, 2010. [09 Ago 2018]. Disponível em: https://censo2010.ibge.gov.br/.

14. Cadastro Nacional de Estabelecimentos de Saúde (CNES). Ficha de Estabelecimento. Brasil, 2018. [15 Ago 2018]. Disponível em: http://cnes.datasus.gov.br/pages/estabelecimentos/consulta.jsp.

15. Instituto Brasileiro de Geografia e Estatística (IBGE). Estatísticas Sociais. Brasil, 2018. [15 Set 2018]. Disponível em: https://www.ibge.gov.br/estatisticasnovoportal/sociais.html.

16. Portal da Saúde (DATASUS). Informações de Saúde (TABNET): Assistência à Saúde. Ministério da Saúde, Brasil, 2008. [08 Ago 2018]. Disponível em: http:/ / www2.datasus.gov.br/DATASUS/index.php?area=0202\&id=19122.

17. Lino PA, Werneck MAF, Lucas SD, Abreu MHNG. Análise da atenção secundária em saúde bucal no estado de Minas Gerais, Brasil. Ciênc. Saúde Colet. 2014; 19(9): 3879-3888.

18. Figueiredo N, Goes PSA. Construção da atenção secundária em saúde bucal: um estudo sobre os centros de especialidades odontológicas em Pernambuco, Brasil. Cad. Saúde Pública. 2009; 25(2): 259-267.

19. Santana VGD, Lima AS, Macedo CLSV, Pimentel FC, Junior JLACA, Martelli JL. Avaliação da evolução do financiamento da assistência odontológica na média complexidade no município de Recife no período de 2000 a 2007. Cad. Saúde Coletiva. 2008; 16(3): 527-543.

20. Informação e Gestão da Atenção Básica (E-Gestor Atenção Básica). Ministério da Saúde, Secretaria de Atenção à Saúde, Departamento de Atenção Básica (DAB). Brasil, 2018. [26 Out 2018]. Disponível em: https://egestorab.saude.gov.br/paginas/acessoPublico/relatorios/relHistor icoCoberturaSB.xhtml\&gt; 
21. Barros AJD, Bertoldi AD. Desigualdades na utilização e no acesso a serviços odontológicos: uma avaliação em nível nacional. Ciênc. Saúde Colet. 2002; 7(4): 709-717.

22. Cortellazzi KL; Balbino EC, Guerra LM, Vazquez FL, Bulgareli JV, Ambrosano GMB et al. Variáveis associadas ao desempenho dos Centros de Especialidades Odontológicas no Brasil.Rev. bras. epidemiol. 2014; 17(4): 978-988.

23. Rangel ML, Limeira RRT, Silva SM, Junior RCM, Ribeiro ILA, Castro RD. Perfil socioeconômico, educacional e de acesso aos serviços ofertados pelos Centros de Especialidades Odontológicas de João Pessoa - Paraíba, Brasil. Revista Família, Ciclos de Vida e Saúde no Contexto Social. 2017; 5(supl.): 1-9.

24. Instituto Nacional do Câncer (INCA). Estimativa 2018: Incidência de Câncer no Brasil. Rio de Janeiro, 2017. [03 Out 2018]. Disponível em: http://www.inca.gov.br/estimativa/2018.

25. Diniz MTM, Oliveira GP. Caracterização geoambiental dos sertões do Seridó Potiguar como subsídio para o planejamento ambiental. Revista Geonorte. 2014; 10(1): 229-233.

Submissão: $28 / 02 / 2019$

Aceitação: 21/10/2019 